\title{
CHIRAL SELECTIVITY IN INTER-REACTANT RECOGNITION AND ELECTRON TRANSFER OF THE OXIDATION OF HORSE HEART CYTOCHROME $C$ BY TRIS-OXALATO-COBALTATE (III)
}

Renat R. Nazmutdinov ${ }^{1 *}$, Michael D. Bronshtein ${ }^{1}$, Tamara T. Zinkicheva ${ }^{1}$, Niels Sthen

Hansen $^{2}$, Jingdong Zhang ${ }^{2}$ and Jens Ulstrup ${ }^{2 * *}$

${ }^{1}$ Kazan National Research Technological University, K. Marx Str., 68, 420015 Kazan, Republic of Tatarstan, Russian Federation

2

Department of Chemistry, Building 207, Technical University of Denmark, 2800 Kongens Lyngby, Denmark

*) nazmutdi@mail.ru; **)ju@kemi.dtu.dk

\section{Rate Equations for Oxidation of Cyt $c\left(\right.$ II) by $R a c-\left[\mathrm{Co}(\mathrm{Ox})_{3}\right]^{3-}$}

We consider oxidation of cyt $c$ (II) by a mixture of $\Lambda$ - and $\Delta-\left[\mathrm{Co}(\mathrm{Ox})_{3}\right]^{3-}$. The fractions of the $\Lambda$ - and $\Delta$-forms are at first arbitrary. As noted in the text, racemization equilibration can be disregarded over the time range of the ET process.

We use the following modified form of eq.(1)

$$
\begin{array}{cll} 
& k_{\mathrm{d} \Lambda} & k_{E T}^{\Lambda} \\
\Lambda-\left[\mathrm{Co}(\mathrm{Ox})_{3}\right]^{3-}+\operatorname{cyt} c(\mathrm{II}) & \square & \mathrm{IP}_{\Lambda} \rightarrow \Lambda-\left[\mathrm{Co}(\mathrm{Ox})_{3}\right]^{4-}+\operatorname{cyt} c(\mathrm{III}) \\
& k_{-\mathrm{d} \Lambda} & \\
& k_{\mathrm{d} \Delta} & k_{E T}^{\Delta} \\
\Delta-\left[\mathrm{Co}(\mathrm{Ox})_{3}\right]^{3-}+\operatorname{cyt} c(\mathrm{II}) & \square & \mathrm{IP}_{\Delta} \rightarrow \Delta-\left[\mathrm{Co}(\mathrm{Ox})_{3}\right]^{4-}+\operatorname{cyt} c(\mathrm{III}) \\
& k_{-\mathrm{d} \Delta}
\end{array}
$$

where $\mathrm{IP}_{\mathrm{X}}, \mathrm{X}=\Lambda, \Delta$ is a short-hand notation for the intermediate ion pair cyt $c(\mathrm{II}) \cdot \mathrm{X}$ $\left[\mathrm{Co}(\mathrm{Ox})_{3}\right]^{3-}$ in the $\Lambda$ - and $\Delta$-form, while $k_{\mathrm{dX}}$ and $k_{-\mathrm{dX}}$ are the rate constants for formation and dissociation of the ion pair. We introduce the following further short-hand notation for the concentrations of the reactants and ion pair 
$\left[\mathrm{X}-\left[\mathrm{Co}(\mathrm{Ox})_{3}\right]^{3-}\right]=a_{\mathrm{X}}, \mathrm{X}=\Lambda, \Delta$

$[\operatorname{cyt} c(\mathrm{II})]=b$

$\left[\mathrm{IP}_{\mathrm{X}}\right]=i p_{\mathrm{X}}, \mathrm{X}=\Lambda, \Delta$

The following kinetic equations then apply

$$
\begin{aligned}
& \frac{d i p_{\Lambda}}{d t}=k_{d \Lambda} a_{\Lambda}\left(b_{t o t}-i p_{\Lambda}-i p_{\Delta}\right)-\left(k_{-d \Lambda}+k_{E T}^{\Lambda}\right) i p_{\Lambda} \\
& \frac{d i p_{\Delta}}{d t}=k_{d \Delta} a_{\Delta}\left(b-i p_{\Lambda}-i p_{\Delta}\right)-\left(k_{-d \Delta}+k_{E T}^{\Delta}\right) i p_{\Delta} \\
& \frac{d b}{d t}=-\left(k_{d \Lambda} a_{\Lambda}+k_{d \Delta} a_{\Delta}\right) b+k_{-d \Lambda} i p_{\Lambda}+k_{-d \Delta} i p_{\Delta}
\end{aligned}
$$

$b_{\text {tot }}$ is the total (known) concentration of cyt $c$ (II). Correction for cyt $c$ (II) bound in the ion pairs are reflected in the first term on the right hand side of the first two equations in eq.(S3). The general solution of this set of equations gives three-exponential kinetics with somewhat voluminous phase (apparent rate constant) expressions with all the elementary rate constants included. Both the experimental data and the theoretical approach, however, relate essentially to the steady state limit which applies sufficiently far from the initial and final stages of the overall ET reaction. We focus on this limit in the following.

Eqs.(S3) reduce to the following form in the steady-state limit.

$$
\begin{aligned}
& \frac{d i p_{\Lambda}}{d t}=k_{d \Lambda} a_{\Lambda}\left(b_{t o t}-i p_{\Lambda}-i p_{\Delta}\right)-\left(k_{-d \Lambda}+k_{E T}^{\Lambda}\right) i p_{\Lambda}=0 \\
& \frac{d i p_{\Delta}}{d t}=k_{d \Delta} a_{\Delta}\left(b-i p_{\Lambda}-i p_{\Delta}\right)-\left(k_{-d \Delta}+k_{E T}^{\Delta}\right) i p_{\Delta}=0
\end{aligned}
$$

Solution of eq.(S4) gives for $i p_{\Lambda}$ and $i p_{\Delta}$ 


$$
\begin{aligned}
& i p_{\Lambda}=\frac{k_{d \Lambda} a_{\Lambda}\left(k_{-d \Delta}+k_{E T}^{\Delta}\right)}{k_{d \Lambda} a_{\Lambda}\left(k_{-d \Delta}+k_{E T}^{\Delta}\right)+k_{d \Delta} a_{\Delta}\left(k_{-d \Lambda}+k_{E T}^{\Lambda}\right)+\left(k_{-d \Lambda}+k_{E T}^{\Lambda}\right)\left(k_{-d \Delta}+k_{E T}^{\Delta}\right)} b_{t o t} \\
& i p_{\Delta}=\frac{k_{d \Delta} a_{\Delta}\left(k_{-d \Lambda}+k_{E T}^{\Lambda}\right)}{k_{d \Lambda} a_{\Lambda}\left(k_{-d \Delta}+k_{E T}^{\Delta}\right)+k_{d \Delta} a_{\Delta}\left(k_{-d \Lambda}+k_{E T}^{\Lambda}\right)+\left(k_{-d \Lambda}+k_{E T}^{\Lambda}\right)\left(k_{-d \Delta}+k_{E T}^{\Delta}\right)} b_{t o t}
\end{aligned}
$$

Eqs.(S5) reduce to the following forms for each isolated enantiomer

$$
i p_{X}=\frac{k_{d X} a_{X}}{k_{d X} a_{X}++\left(k_{-d X}+k_{E T}^{X}\right)} b_{t o t}, \mathrm{X}=\Lambda, \Delta
$$

or, if $k_{\mathrm{dX}}, k_{-\mathrm{dX}} \gg>k_{E T}^{X}, \mathrm{X}=\Lambda, \Delta$, then

$$
i p_{X}=\frac{K_{X} a_{X}}{1+K_{X} a_{X}} b_{t o t}
$$

where $K_{\mathrm{X}}$ is the equilibrium constant for ion pair formation. This form was used in the data analysis for the pure enantiomers.

Introducing the notation

$a_{\mathrm{tot}}=a_{\Lambda}+a_{\Delta}=P_{\Lambda} a_{\mathrm{tot}}+P_{\Delta} a_{\mathrm{tot}} ; \quad P_{\Lambda}+P_{\Delta}=1$

the observed rate constant for a $\Lambda / \Delta$ mixture of general composition, $k_{\text {obs }}$ becomes

$$
k_{o b s}=\frac{k_{E T}^{\Lambda} K_{\Lambda} P_{\Lambda} a_{\Lambda}}{1+K_{\Lambda} a_{\Lambda}}+\frac{k_{E T}^{\Delta} K_{\Delta}\left(1-P_{\Lambda}\right) a_{\Delta}}{1+K_{\Delta} a_{\Delta}}
$$

For the racemic mixture, $P_{\Lambda}=P_{\Delta}=1 / 2$, or

$$
k_{o b s}=\frac{k_{E T}^{\Lambda} K_{\Lambda} a_{t o t}}{2+K_{\Lambda} a_{t o t}}+\frac{k_{E T}^{\Delta} K_{\Delta} a_{t o t}}{2+K_{\Delta} a_{t o t}}
$$


In the data analysis for the pure enantiomers, values of $k_{E T}^{\Lambda}$ and $k_{E T}^{\Delta}$ were obtained for high Co(III) concentrations, for which $K_{\mathrm{X}} a_{\mathrm{X}}>1$ and values of $k_{E T}^{X} \cdot K_{\mathrm{X}}$, i.e. of $K_{\mathrm{X}}, \mathrm{X}=\Lambda, \Delta$ at low $\mathrm{Co}(\mathrm{III})$ concentrations for which $K_{\mathrm{X}} a_{\mathrm{X}}<<1$. The corresponding values which can be obtained for the 1:1 racemic mixture are $\frac{1}{2}\left(k_{E T}^{\Lambda}+k_{E T}^{\Delta}\right)$ and $\frac{1}{2}\left(k_{E T}^{\Lambda} K_{\Lambda}+k_{E T}^{\Delta} K_{\Delta}\right)$, respectively, as given in Table 1.
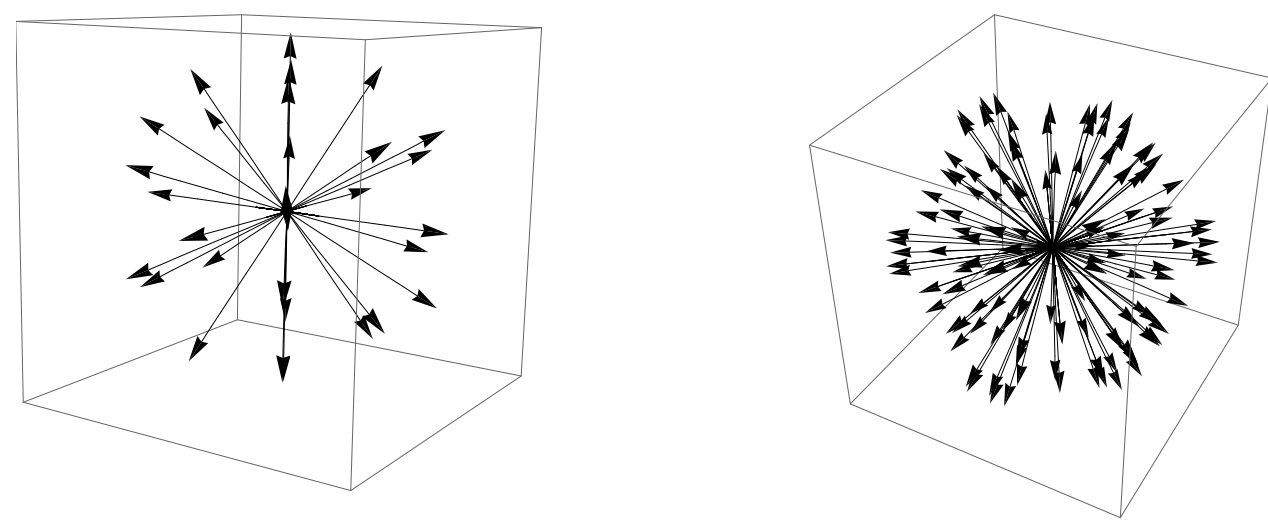

Figure S1. Sketches demonstrating 30 (left) and 120 (right) rotational axes directed from the central atom of $\left[\mathrm{Co}(\mathrm{Ox})_{3}\right]^{3-}$.

Table S1. Co-O bond lengths calculated for $\left[\mathrm{Co}(\mathrm{Ox})_{3}\right]^{3-}$ and $\left[\mathrm{Co}(\mathrm{Ox})_{3}\right]^{4-}$. The numbers of the corresponding $\mathrm{O}$ atoms are given in parentheses.

\begin{tabular}{|c|c|c|c|}
\hline & $\begin{array}{c}{\left[\mathrm{Co}(\mathrm{Ox})_{3}\right]^{3-}} \\
(\text { singlet })\end{array}$ & $\begin{array}{c}{\left[\mathrm{Co}(\mathrm{Ox})_{3}\right]^{4-}} \\
\text { (doublet) }\end{array}$ & $\begin{array}{c}{\left[\mathrm{Co}(\mathrm{Ox})_{3}\right]^{4-}} \\
(\text { doublet })\end{array}$ \\
\hline $\mathrm{r}(\mathrm{Co}-\mathrm{O}), \AA$ & 1.906 & $2.1075(3) ;$ & $1.936(2) ; 2.096(2) ;$ \\
& & $2.1305(3)$ & $2.114(2)$ \\
\hline
\end{tabular}

Table S2. Parameters of the Lennard-Jones (LJ) potential used in the calculations.

\begin{tabular}{|c|c|c|c|c|c|c|}
\hline & $\mathbf{H}$ & $\mathbf{C}$ & $\mathbf{N}$ & $\mathbf{O}$ & $\mathbf{S}$ & $\mathbf{K}$ \\
\hline$\varepsilon, \mathrm{eV}$ & 0.00104 & 0.0034 & 0.00303 & 0.0052 & 0.015 & 0.01401 \\
\hline$\sigma, \AA$ & 2.68 & 4 & 4 & 3.4 & 4 & 3.51 \\
\hline
\end{tabular}


Table S3. Some details of the grid construction in the calculations of the kinetic parameters.

\begin{tabular}{|l|c|c|}
\hline kinetic parameters & $K_{X}$ & $\kappa_{e l}^{X}$ \\
\hline grid step, $\AA$ & 0.64 & 1. \\
\hline number of rotational axes & 120 & 30 \\
\hline number of rotations around each axis & 11 & 11 \\
\hline
\end{tabular}

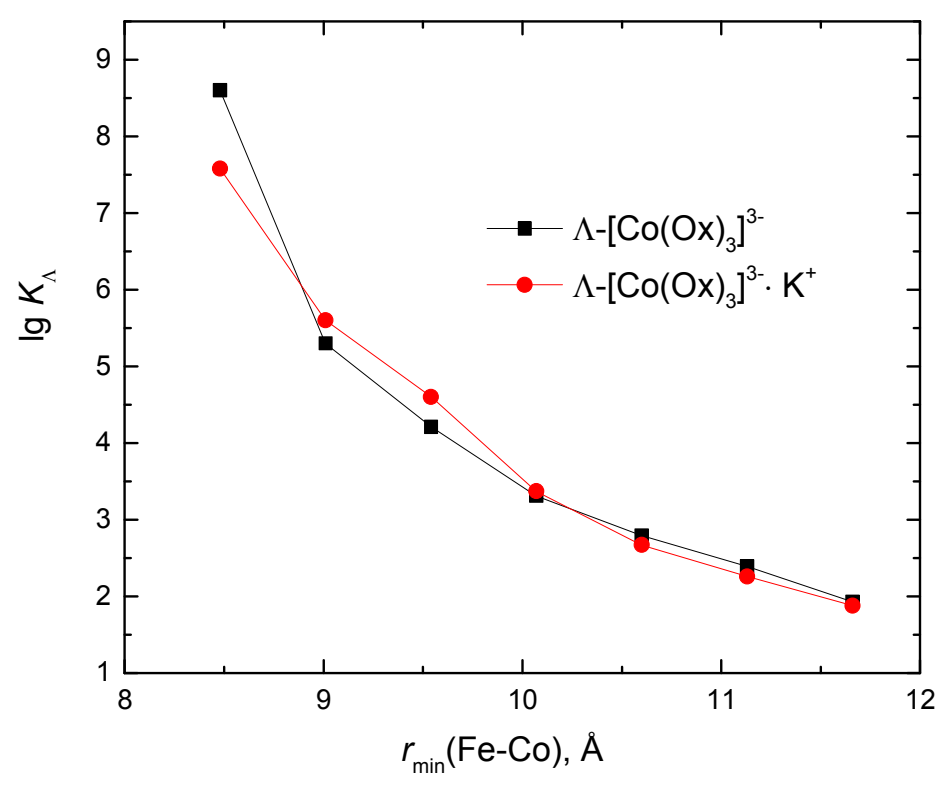

Figure S2. Dependence of $K_{\Delta}$ on $r_{\min }(\mathrm{Fe}-\mathrm{Co})$ calculated for singlet cyt $c$ heme and the $\Lambda$ $\left[\mathrm{Co}(\mathrm{Ox})_{3}\right]^{3-}$ and $\Lambda-\left[\mathrm{Co}(\mathrm{Ox})_{3}\right]^{3-} \cdot \mathrm{K}^{+}$complexes. 


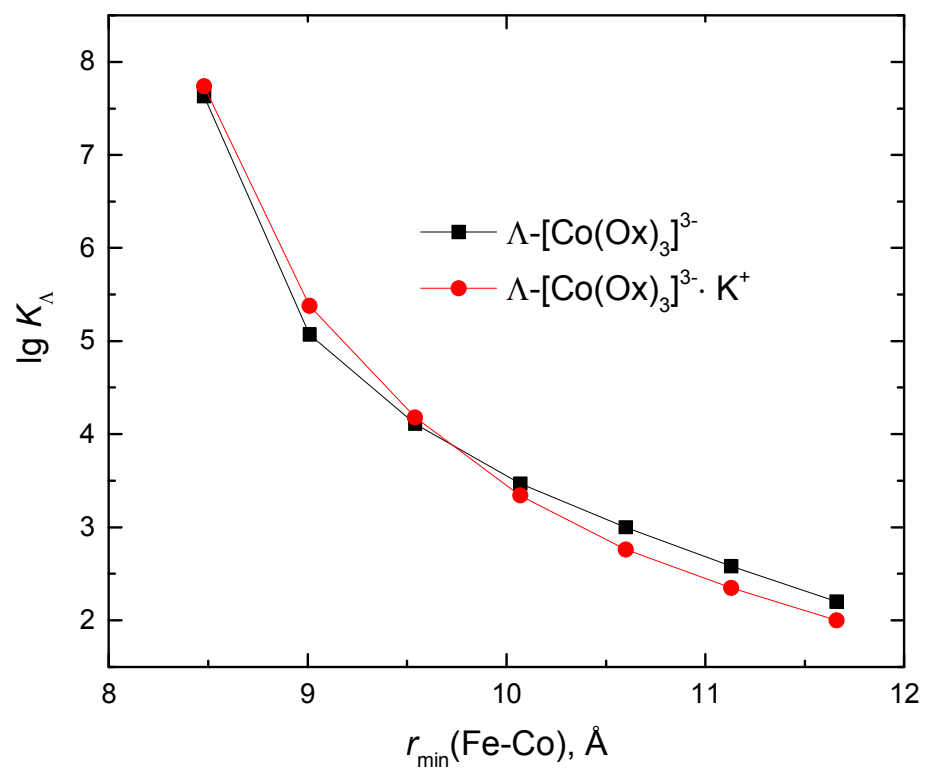

Figure S3. Dependence of $K_{\Lambda}$ on $r_{\min }(\mathrm{Fe}-\mathrm{Co})$ calculated for triplet cyt $c$ heme and the $\Lambda$ $\left[\mathrm{Co}(\mathrm{Ox})_{3}\right]^{3-}$ and $\Lambda-\left[\mathrm{Co}(\mathrm{Ox})_{3}\right]^{3-} \cdot \mathrm{K}^{+}$complexes.

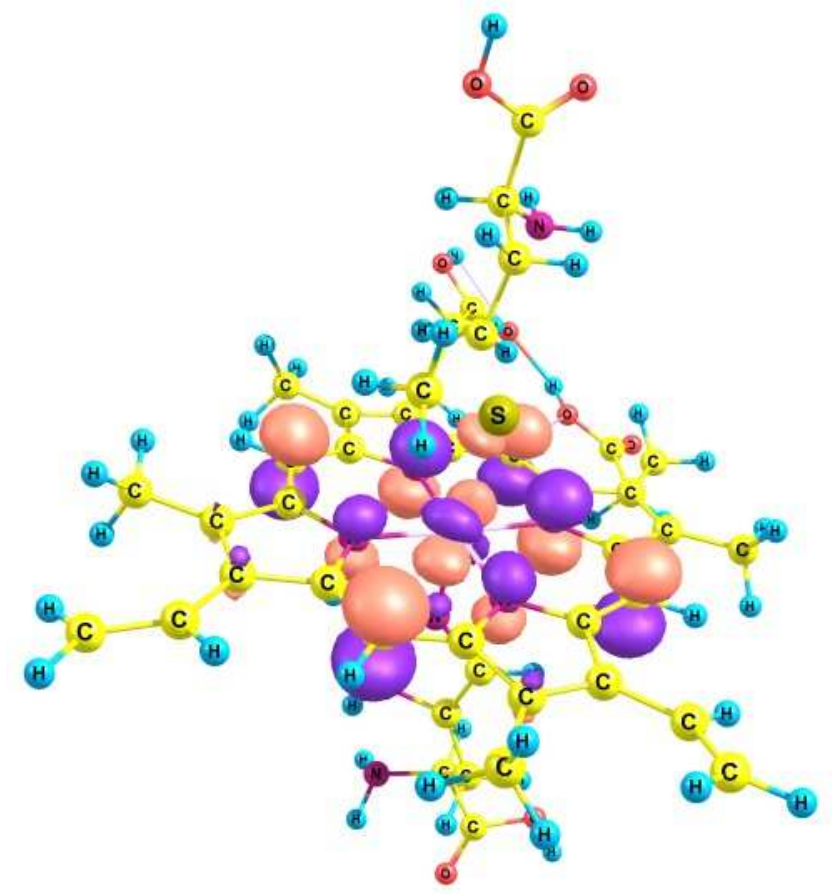

(a) 


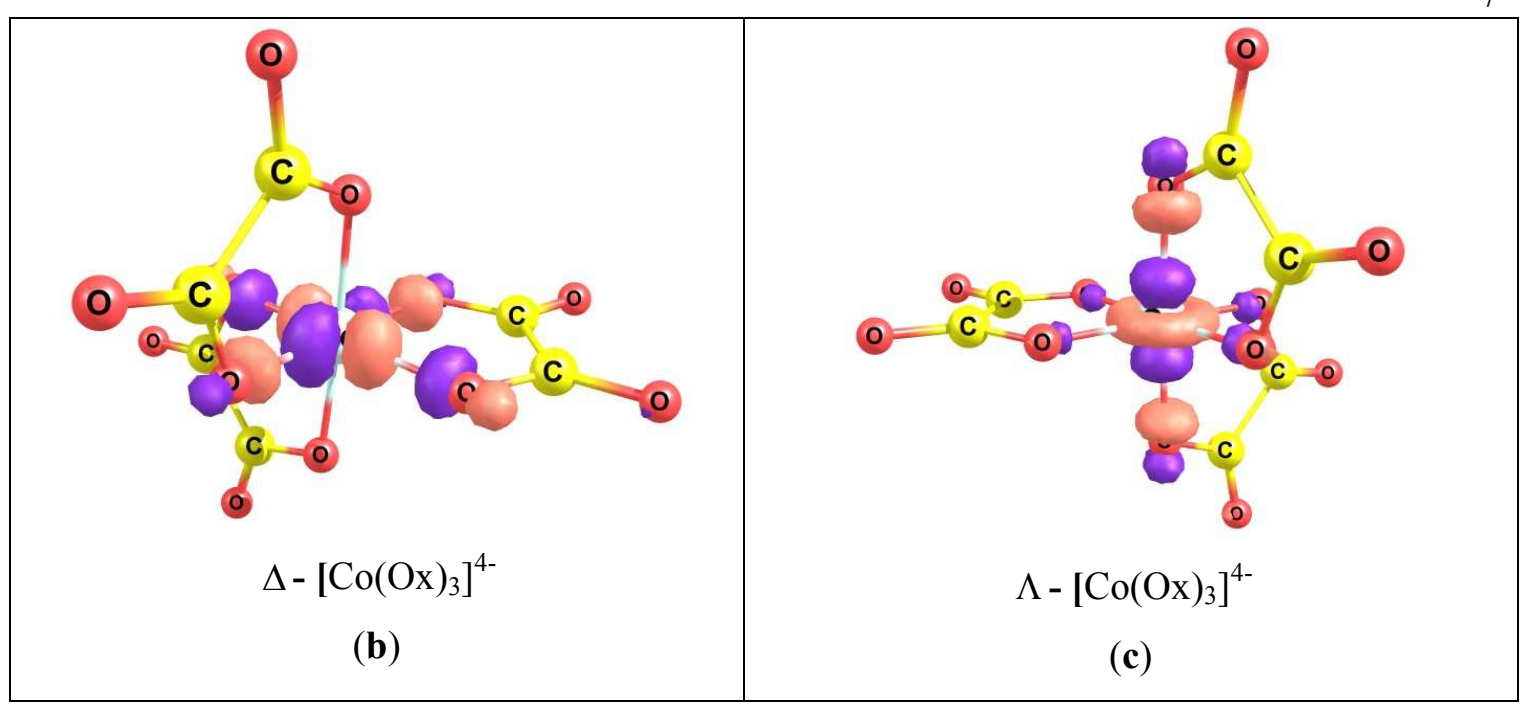

Figure S4. Three-dimensional representations of the Highest Occupied Molecular Orbitals of the reduced cyt $c$ heme group (a), $\Delta-\left[\mathrm{Co}(\mathrm{Ox})_{3}\right]^{4-}$ (b) and $\Lambda-\left[\mathrm{Co}(\mathrm{Ox})_{3}\right]^{4-}$ (c). The relaxed geometry of oxidized $\left[\mathrm{Co}(\mathrm{Ox})_{3}\right]^{3-}$ was used in calculations.

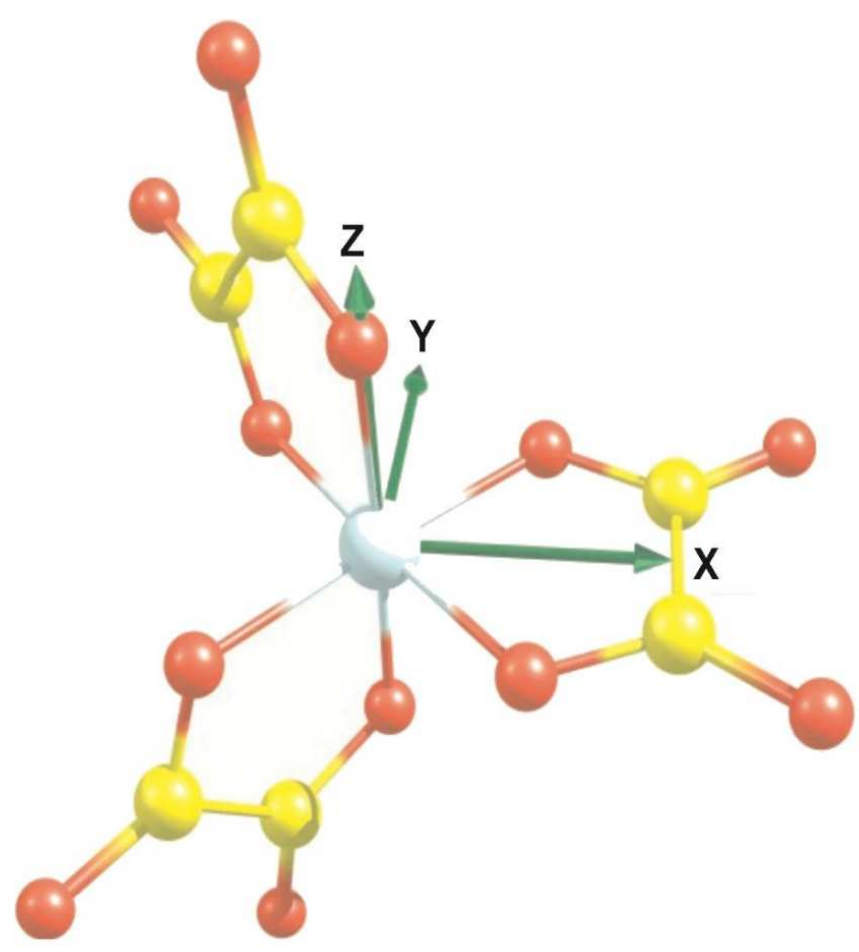


Figure S5. Local coordinate system used in the DFT calculations of $\left[\mathrm{Co}(\mathrm{Ox})_{3}\right]^{3-/ 4-}$.

Table S4. Optimized geometry of the model cyt $c$ heme group.

\begin{tabular}{|c|c|c|c|}
\hline \multirow[t]{2}{*}{ Atom } & \multicolumn{3}{|c|}{ Cartesian coordinates, $\AA$} \\
\hline & $x$ & $y$ & $z$ \\
\hline $\mathrm{c}$ & -2.42175 & 1.02995 & -2.21764 \\
\hline $\mathrm{h}$ & -1.79309 & -0.15326 & -5.27005 \\
\hline $\mathrm{c}$ & -2.58145 & -0.20178 & -4.50567 \\
\hline $\mathrm{c}$ & -0.27201 & -1.74955 & -3.09801 \\
\hline $\mathrm{c}$ & -0.95079 & -0.69048 & -2.4933 \\
\hline $\mathrm{h}$ & 5.10381 & -3.25995 & 5.11181 \\
\hline $\mathrm{c}$ & 3.94749 & -5.58998 & -3.65001 \\
\hline $\mathrm{c}$ & 3.54696 & -4.97318 & -2.50727 \\
\hline $\mathrm{c}$ & 2.39916 & -3.09613 & -1.14229 \\
\hline $\mathrm{c}$ & 3.09479 & -2.4584 & 1.16351 \\
\hline $\mathrm{fe}$ & 0.7431 & -0.81713 & 0.07322 \\
\hline $\mathrm{n}$ & 2.13499 & -1.47612 & 1.39349 \\
\hline $\mathrm{n}$ & 0.09407 & 0.52248 & 1.44489 \\
\hline $\mathrm{n}$ & -0.68622 & -0.19374 & -1.22048 \\
\hline $\mathrm{n}$ & 1.34053 & -2.2111 & -1.27727 \\
\hline $\mathrm{c}$ & 3.21954 & -3.19689 & -0.01601 \\
\hline $\mathrm{h}$ & 4.02259 & -3.92428 & -0.05253 \\
\hline $\mathrm{c}$ & 2.53567 & -3.92888 & -2.34818 \\
\hline $\mathrm{h}$ & 4.03968 & -5.29134 & -1.58759 \\
\hline $\mathrm{h}$ & 4.70851 & -6.36627 & -3.621 \\
\hline $\mathrm{h}$ & 3.55651 & -5.33393 & -4.62929 \\
\hline $\mathrm{c}$ & 1.52142 & -3.53052 & -3.21316 \\
\hline $\mathrm{c}$ & 1.18485 & -4.09009 & -4.56924 \\
\hline $\mathrm{h}$ & 0.15939 & -3.84687 & -4.868 \\
\hline $\mathrm{h}$ & 1.28317 & -5.18341 & -4.57479 \\
\hline $\mathrm{h}$ & 1.85511 & -3.70234 & -5.35146 \\
\hline $\mathrm{c}$ & 0.79858 & -2.45727 & -2.53801 \\
\hline $\mathrm{c}$ & 2.35439 & -1.02628 & 2.68546 \\
\hline $\mathrm{c}$ & 3.94621 & -2.63599 & 2.33795 \\
\hline $\mathrm{c}$ & 5.112 & -3.58257 & 2.43455 \\
\hline $\mathrm{h}$ & 5.49294 & -3.86222 & 1.44628 \\
\hline $\mathrm{h}$ & 4.84311 & -4.51252 & 2.9583 \\
\hline
\end{tabular}


9

\begin{tabular}{|c|c|c|c|}
\hline $\mathrm{h}$ & 5.93898 & -3.12547 & 2.99291 \\
\hline $\mathrm{c}$ & 3.47909 & -1.74922 & 3.30128 \\
\hline $\mathrm{c}$ & 3.96558 & -1.49206 & 4.65628 \\
\hline $\mathrm{h}$ & 3.64456 & -0.54691 & 5.09654 \\
\hline $\mathrm{c}$ & 4.75778 & -2.28122 & 5.42803 \\
\hline $\mathrm{h}$ & 5.05693 & -1.96006 & 6.42304 \\
\hline $\mathrm{c}$ & 1.61196 & -0.02334 & 3.31737 \\
\hline $\mathrm{h}$ & 1.86436 & 0.21818 & 4.34439 \\
\hline $\mathrm{c}$ & 0.5636 & 0.69454 & 2.74083 \\
\hline $\mathrm{c}$ & -0.93113 & 1.44525 & 1.2897 \\
\hline $\mathrm{c}$ & -0.19346 & 1.74785 & 3.42997 \\
\hline $\mathrm{c}$ & -1.12418 & 2.21595 & 2.527 \\
\hline $\mathrm{c}$ & 0.04023 & 2.17797 & 4.8544 \\
\hline $\mathrm{h}$ & -0.08782 & 1.34148 & 5.5558 \\
\hline $\mathrm{h}$ & -0.6574 & 2.96842 & 5.15235 \\
\hline $\mathrm{h}$ & 1.05815 & 2.56792 & 4.9978 \\
\hline $\mathrm{h}$ & -0.59205 & -2.03369 & -4.0954 \\
\hline $\mathrm{c}$ & -2.0363 & 0.06467 & -3.12643 \\
\hline $\mathrm{h}$ & -3.03699 & -1.20013 & -4.57672 \\
\hline $\mathrm{h}$ & -3.35071 & 0.52888 & -4.78013 \\
\hline $\mathrm{c}$ & -1.57629 & 0.8526 & -1.03159 \\
\hline $\mathrm{c}$ & -3.49293 & 2.08195 & -2.36235 \\
\hline $\mathrm{c}$ & -1.69418 & 1.6145 & 0.13368 \\
\hline $\mathrm{h}$ & -2.44923 & 2.39226 & 0.14761 \\
\hline $\mathrm{c}$ & -2.2052 & 3.25108 & 2.72291 \\
\hline $\mathrm{h}$ & -3.16274 & 2.82185 & 2.4043 \\
\hline $\mathrm{h}$ & -2.30874 & 3.48686 & 3.78808 \\
\hline $\mathrm{h}$ & -3.67111 & 2.29345 & -3.42449 \\
\hline $\mathrm{h}$ & -3.17142 & 3.01872 & -1.89409 \\
\hline $\mathrm{c}$ & -4.84784 & 1.64506 & -1.7067 \\
\hline $\mathrm{h}$ & -5.23393 & 0.74966 & -2.19962 \\
\hline $\mathrm{h}$ & -4.67091 & 1.408 & -0.65133 \\
\hline $\mathrm{c}$ & -1.98076 & 4.5919 & 1.93387 \\
\hline $\mathrm{h}$ & -1.24791 & 4.42136 & 1.13631 \\
\hline $\mathrm{h}$ & -1.59215 & 5.37502 & 2.59049 \\
\hline $\mathrm{c}$ & -3.2529 & 5.10624 & 1.28091 \\
\hline o & -3.80168 & 6.20027 & 1.48935 \\
\hline o & -3.74148 & 4.17792 & 0.38514 \\
\hline
\end{tabular}




\begin{tabular}{|c|c|c|c|}
\hline $\mathrm{h}$ & -4.58657 & 4.38415 & -0.10665 \\
\hline $\mathrm{c}$ & -5.88048 & 2.74184 & -1.78836 \\
\hline o & -5.82345 & 3.86579 & -1.24158 \\
\hline o & -6.96074 & 2.41578 & -2.58208 \\
\hline $\mathrm{h}$ & -7.60461 & 3.16123 & -2.62629 \\
\hline $\mathrm{c}$ & 4.5705 & 4.35437 & -1.55172 \\
\hline $\mathrm{h}$ & 3.56024 & 4.76615 & -1.44536 \\
\hline $\mathrm{c}$ & 5.56432 & 5.44379 & -1.12956 \\
\hline $\mathrm{c}$ & 4.69798 & 3.13329 & -0.58823 \\
\hline $\mathrm{h}$ & 5.69745 & 2.69137 & -0.72927 \\
\hline $\mathrm{h}$ & 4.64369 & 3.48569 & 0.44631 \\
\hline $\mathrm{n}$ & 4.76931 & 3.94018 & -2.95634 \\
\hline $\mathrm{h}$ & 5.74758 & 3.7194 & -3.15254 \\
\hline $\mathrm{h}$ & 4.43652 & 4.6363 & -3.62381 \\
\hline o & 5.20174 & 6.02975 & 0.07048 \\
\hline $\mathrm{h}$ & 5.85096 & 6.71521 & 0.35054 \\
\hline o & 6.58619 & 5.77032 & -1.75085 \\
\hline $\mathrm{c}$ & 3.62483 & 2.10708 & -0.8119 \\
\hline $\mathrm{n}$ & 3.23221 & 1.70558 & -2.09293 \\
\hline $\mathrm{c}$ & 2.86928 & 1.34332 & 0.06312 \\
\hline $\mathrm{c}$ & 2.2687 & 0.7386 & -1.96507 \\
\hline $\mathrm{h}$ & 1.77547 & 0.25396 & -2.78966 \\
\hline $\mathrm{n}$ & 2.03259 & 0.49586 & -0.66833 \\
\hline $\mathrm{h}$ & 2.87114 & 1.34048 & 1.13979 \\
\hline $\mathrm{h}$ & 3.60271 & 2.17023 & -2.92267 \\
\hline $\mathrm{c}$ & -0.55038 & -4.17764 & 0.54307 \\
\hline $\mathrm{h}$ & 0.49527 & -4.40769 & 0.75439 \\
\hline $\mathrm{h}$ & -0.7176 & -4.1916 & -0.53636 \\
\hline $\mathrm{h}$ & -1.20187 & -4.89163 & 1.05342 \\
\hline $\mathrm{s}$ & -0.85614 & -2.44012 & 1.20776 \\
\hline $\mathrm{c}$ & -2.60849 & -2.14278 & 0.54851 \\
\hline $\mathrm{h}$ & -2.60197 & -2.37941 & -0.52106 \\
\hline $\mathrm{h}$ & -2.77774 & -1.07174 & 0.66266 \\
\hline $\mathrm{c}$ & -3.66705 & -2.95241 & 1.30937 \\
\hline $\mathrm{h}$ & -3.49766 & -4.02788 & 1.18249 \\
\hline $\mathrm{h}$ & -3.59835 & -2.73057 & 2.38447 \\
\hline $\mathrm{c}$ & -5.10643 & -2.62253 & 0.81347 \\
\hline $\mathrm{h}$ & -5.14376 & -2.80828 & -0.26789 \\
\hline
\end{tabular}




\begin{tabular}{|l|l|l|l|}
\hline $\mathrm{n}$ & -5.44526 & -1.22137 & 1.06731 \\
$\mathrm{c}$ & -6.09612 & -3.60393 & 1.46428 \\
$\mathrm{o}$ & -6.93312 & -3.33387 & 2.33754 \\
$\mathrm{o}$ & -5.95281 & -4.88988 & 0.95508 \\
$\mathrm{~h}$ & -6.57875 & -5.51456 & 1.38831 \\
$\mathrm{~h}$ & -5.39698 & -0.96822 & 2.05309 \\
$\mathrm{~h}$ & -6.33039 & -0.92809 & 0.65913 \\
\hline
\end{tabular}

Table 5S. Optimized geometry of $\Delta-\left[\mathrm{Co}(\mathrm{Ox})_{3}\right]^{3-}$.

\begin{tabular}{|c|c|c|c|}
\hline \multirow{2}{*}{ Atom } & \multicolumn{3}{|c|}{ Cartesian coordinates, $\AA$} \\
\cline { 2 - 4 } & $x$ & $y$ & $z$ \\
\hline Co & 0. & 0.00091 & 0. \\
O & 0.75362 & 1.39493 & 1.0588 \\
O & -0.75362 & 1.39493 & -1.0588 \\
C & -0.45633 & 2.60989 & -0.62976 \\
O & -0.85161 & 3.67718 & -1.16041 \\
C & 0.45633 & 2.60989 & 0.62976 \\
O & 0.85161 & 3.67718 & 1.16041 \\
O & 1.58427 & -0.04594 & -1.05939 \\
O & 0.83023 & -1.34713 & 1.06094 \\
C & 2.03247 & -1.69546 & 0.63537 \\
O & 2.76322 & -2.56253 & 1.17497 \\
C & 2.4848 & -0.91517 & -0.63208 \\
O & 3.60231 & -1.11623 & -1.16889 \\
O & -0.83023 & -1.34713 & -1.06094 \\
O & -1.58427 & -0.04594 & 1.05939 \\
C & -2.4848 & -0.91517 & 0.63208 \\
O & -3.60231 & -1.11623 & 1.16889 \\
C & -2.03247 & -1.69546 & -0.63537 \\
O & -2.76322 & -2.56253 & -1.17497 \\
\hline
\end{tabular}


Table 6S. Optimized geometry of $\Lambda-\left[\mathrm{Co}(\mathrm{Ox})_{3}\right]^{3-}$.

\begin{tabular}{|c|c|c|c|}
\hline \multirow{2}{*}{ Atom } & \multicolumn{3}{|c|}{ Cartesian coordinates, $\AA$} \\
\cline { 2 - 4 } & $x$ & $y$ & $z$ \\
\hline Co & -0.00484 & -0.01062 & -0.00247 \\
O & 0.94233 & 1.2605 & -1.05977 \\
O & -0.56138 & 1.47756 & 1.0497 \\
C & -0.08107 & 2.63585 & 0.62995 \\
O & -0.30417 & 3.74669 & 1.17219 \\
C & 0.81648 & 2.50605 & -0.63432 \\
O & 1.35222 & 3.50665 & -1.17167 \\
O & -1.57101 & 0.16177 & -1.07517 \\
O & -1.02637 & -1.22346 & 1.05535 \\
C & -2.27 & -1.38118 & 0.6345 \\
O & -3.12617 & -2.12059 & 1.18013 \\
C & -2.59674 & -0.54982 & -0.63916 \\
O & -3.73164 & -0.57917 & -1.17603 \\
O & 0.64376 & -1.4656 & -1.05056 \\
O & 1.55175 & -0.26553 & 1.06852 \\
C & 2.3426 & -1.23396 & 0.63855 \\
O & 3.4313 & -1.56343 & 1.17093 \\
C & 1.79429 & -1.95863 & -0.62392 \\
O & 2.41154 & -2.91328 & -1.15946 \\
\hline
\end{tabular}

\section{Appendix A}

In our model the quantities $K_{X}\left(W_{R}^{X}\right)$ and $\kappa_{e l}^{X}$ are statistically averaged over a large number of inter-reactant distances and mutual orientations, 
$\kappa_{e l}^{X}=\sum_{j} f_{j} \kappa_{e l}^{X,(j)}$ and $\quad W_{R}^{X}=\sum_{j} f_{j} W_{R}^{X,(j)}$

where

$$
f_{j}=\frac{\exp \left[-\frac{W_{R}^{X,(j)}}{k_{B} T}\right]}{\sum_{j} \exp \left[-\frac{W_{R}^{X,(j)}}{k_{B} T}\right]}
$$

and $\mathrm{j}$ denotes a certain configuration.

\section{Appendix B}

The $E_{\text {in }}$ values were calculated as the difference in total energies of reactants in vacuum: $E_{\text {in }}($ red $)=E_{\text {tot,noneq }}($ red $)-E_{\text {tot,eq }}($ red $)$, where $E_{\text {tot,noneq }}($ red $)$ is the reactant total energy in the reduced state calculated for the non-equilibrium geometry corresponding to the oxidized form. Similarly, $E_{\text {in }}(\mathrm{ox})=E_{\text {tot,noneq }}(\mathrm{ox})-E_{\text {tot,eq }}(\mathrm{ox})$, where $E_{\text {tot,noneq }}(\mathrm{ox})$ is the reactant total energy in the oxidized state, calculated for the non-equilibrium geometry corresponding to the reduced form. This simple approach is correct, because the contribution of high-frequency (quantum) vibrational modes to the inner-sphere reorganization is very small.

\section{Appendix C}

Eq.(9) has been re-written as follows,

$W_{R}^{X,(j)} \approx \sum_{k, i} U_{L D}\left(R_{k i}\right)+\frac{1}{2 \varepsilon_{e f f}^{s t a t}} \sum_{k, i} \frac{q_{k} q_{i}}{R_{k i}}$.

The LJ potentials $U_{i j}\left(R_{i j}\right)$ are represented in the form

$$
U_{L D}\left(R_{k i}\right)=\varepsilon_{k i}\left[\left(\frac{\sigma_{k i}}{R_{k i}}\right)^{12}-2\left(\frac{\sigma_{k i}}{R_{k i}}\right)^{6}\right] .
$$


The non-diagonal parameters $\varepsilon_{i j}$ and $\sigma_{i j}$ were calculated according to the commonly adopted rules:

$$
\varepsilon_{k i}=\sqrt{\varepsilon_{k k} \varepsilon_{i i}} \text { and } \sigma_{k i}=\frac{1}{2}\left(\sigma_{k k}+\sigma_{i i}\right)
$$

LJ parameters were taken from Refs.S1-S3 (see also Table 2S). The atomic charges were calculated using the ChelpG method $^{\mathrm{S} 4}$ which provides the best molecular electrostatic potential fit. The same method was employed to $\kappa_{e l}^{X}$ (see below).

[S1] Strader, M.; Feller, S.E. A flexible all-atom model of DMSO for molecular dynamics simulations. J. Phys. Chem. A. 2002, 106, 1074-1080.

[S2] Chen, L.J.; Yin, D.; MacKerell, A.D. Combined ab initio/empirical approach for optimization of LJ parameters for polar-neutral compounds. J. Comp. Chem. 2002, 23, 199-213.

[S3] Hess, B.; van der Vegt, N.F.A. Cation specific binding with protein surface charges. PNAS 2009, 106, 13296-1330.

[S4] Breneman, C.M.; Wiberg, K.B. Determining atom-centered monopoles from molecular electrostatic potentials. The need for high sampling density in foramide conformational analysis. J. Comput. Chem.. 1990, 11, 361-373.

\section{Appendix D}

The heme group was first located in the centre of a $2 \times 2 \times 2 \mathrm{~nm}^{3}$ cube consisting of a large number of smaller cubic cells with the edge length $0.64 \AA$ (for $K$ ) and $1 \AA$ (for $\kappa_{\mathrm{el}}$ ). The $\left[\mathrm{Co}(\mathrm{Ox})_{3}\right]^{3-}$ complex was then positioned successively in the centre of each of the small cells, by altogether 11 rotations over 120 or 30 different axes (in the calculation of $K$ and $\kappa_{\mathrm{el}}$, respectively) directed from the Co atom (see Fig.1S). A smaller grid was used to estimate averaged $\kappa_{\mathrm{el}}$ values as such calculations are quite computer time demanding. Some technical details are compiled in Table S3. The rotated molecular $\left[\mathrm{Co}(\mathrm{Ox})_{3}\right]^{3-}$ orbitals are transformed using the Wigner matrix. For convenience of further analysis the grid array was rearranged in concentric spheres with different radii around the heme centre. A special geometrical 
constraint which prevents small (non-physical) interatomic distances was used in the grid construction.

Calculation of three-centre integrals was performed using an original scheme based on a routine of sorting the most important non-diagonal contributions to the integral. This routine makes calculations possible within a reasonable time scale even for large grids. All calculations of $K_{X}$ and $\kappa_{e l}^{X}$ were based on a code written by the Mathematica 9 program suite. 